\title{
Snakes in the garden: an analysis of reptiles "rescued" by community-based wildlife carers
}

\author{
Richard Shine*, Jennifer Koenig \\ Biological Sciences A08, University of Sydney, NSW 2006 Australia
}

Received 16 November 2000; received in revised form 13 March 2001; accepted 23 March 2001

\begin{abstract}
Stimulated by animal-welfare concerns, community programs to "rescue" urban wildlife generate many interactions between humans and wildlife. Such rescue activities (1) may have direct ecological effects (by modifying mortality patterns of wildlife, or geographic distributions at the local level), and (2) may provide valuable information on local abundance and distribution of taxa, the nature of threats to urban wildlife, and biological attributes of poorly-known species. We examine these issues for reptiles rescued by community-based animal-welfare groups in south-eastern Australia. Records gathered by the Wildlife Information and Rescue Service over a 10-year period (1989-1998) in New South Wales quantify rates, determinants and outcomes of reptile rescues. Despite their scarcity in urban habitats, snakes (11,067 records) were represented almost as often as lizards (11,108). Typically, rescued lizards were large in size or snake-like in appearance. Most reptiles (especially snakes) were rescued not because of injury, but because the local residents wanted them removed. Thus, very large numbers of these animals were translocated to release sites. Of the injured animals, small-bodied reptile species (and juveniles of larger species) were frequent victims of attacks by domestic cats and dogs. Larger reptiles were more often injured by motor vehicles. Capture rates for all species were highest in warmer months, and especially on days with dry, warm weather. These temporal patterns seem to be due to reptile biology not human behaviour. The rapid increase in animal-rescue activities indicates that potential ecological effects (especially, arising from translocation of "problem" wildlife) deserve further study. At the same time, such community groups can provide valuable information for ecologists. (C) 2001 Elsevier Science Ltd. All rights reserved.
\end{abstract}

Keywords: Lizard; Mortality; New South Wales; Relocation; Snake; Sydney

\section{Introduction}

The last few decades have seen the emergence of a dichotomy in approaches to wildlife management. One stream consists of scientists trained in ecology and conservation biology, who frame their research projects in terms of population viability. The other stream involves members of animal-welfare groups, generally with little scientific training. These groups focus on the fate of individual animals, and their most common direct interaction with wildlife involves animal "rescue" (Kaplan, 1999; Underhill et al., 1999). Such groups encourage the local community to alert them to the presence of "problem" wildlife. Then, volunteers go out and collect the animal. Many of these rescued animals

* Corresponding author. Tel.: + 61-2-9351-3772; fax: + 61-2-93515609.

E-mail address: rics@bio.usyd.edu.au (R. Shine). have been injured by humans, or their domestic pets or motor vehicles (Philcox et al., 1999). Some are nursed back to health, whereas others do not survive. Other "rescues" involve uninjured animals that are perceived as being at risk, often because their habitat is being destroyed in the course of urban development. Yet other taxa are seen as a potential risk to local people. For some types of animals, such as snakes, all three categories are important.

Traditionally, these two major streams - professional ecologists and wildlife rescuers - have displayed little co-operation with each other. Indeed, their relationships have often been acrimonious, and many practitioners on either side of the debate have emphasised fundamental differences between the two approaches (Kaplan, 1999). Nonetheless, there are also broad areas of overlap. The activities of "animal rescue" organisations are relevant to professional ecologists for at least two reasons: 
(1) Direct ecological impact. - These interactions between people and wildlife may modify patterns of mortality in natural populations, especially in areas close to cities and towns. Shifts in mortality rates, or the distribution of that mortality among species, sexes and age classes, may have important ecological consequences (e.g. Underhill et al., 1999). Additionally, wildlife rescue groups translocate many animals, moving them from "unsuitable" to "suitable" habitats. There is thus an obvious potential for shifts in local abundance and distribution, and changes in age and sex structure. Also, the transfer of pathogens and of novel genetic material could affect recipient populations (Madsen et al., 1999; Berish et al., 2000).

(2) Acquisition of information. - Many of the community-based groups maintain careful records of their activities. Although the charters of some groups specifically prohibit the use of rescued wildlife for research purposes (Kaplan, 1999), this restriction is less likely to be applied to the data than to the animals themselves. Thus, such data may provide valuable information on: (1) local abundance and distribution of taxa; (2) the nature of threats to urban wildlife populations; and (3) basic biological topics such as spatial and temporal patterns in activity and age structure of poorly-known taxa. Such information may assist conservation planning for rare and vulnerable species, or reduce threats to the public posed by dangerous species such as venomous snakes. Unfortunately, the nature of wildlife rescue may introduce massive biases to such data. For example, some species will be regarded as higher priority for rescue than others. Hence, any use of such data needs to accommodate the nature and magnitude of such biases.

The activities of "animal rescue" groups also are relevant to professional ecologists in other, less direct ways. For many urban-dwellers, community "animal rescue" groups offer the most direct contact with wildlife. The policies of these groups may thus modify public attitudes to management, and hence place strong social and political pressures on the authorities responsible for wildlife management. Although difficult to quantify, such processes may restrict the range of options open to professional managers.

The significance of each of these facets will clearly show strong geographic and taxonomic influences. Nonetheless, cities are expanding worldwide, increasing the encounters between humans and other animals. In this paper, we explore a case study of "animal rescue" interactions between humans and wildlife. To do so, we take advantage of the fact that the larger wildlife rescue organisations record the numbers of animals collected, the reason for their collection, and the animals' subsequent fate (see earlier). We analyse a large data set of this kind to identify and interpret broad trends in the numbers, circumstances and outcomes of human encounters with snakes and lizards in a region of eastern Australia.

\section{Materials and methods}

The New South Wales Wildlife Information and Rescue Service (WIRES) is a volunteer-based organisation that rescues injured or abandoned native wildlife in New South Wales (NSW), Australia. We collated all records of snake and lizard rescues from the WIRES data-base for New South Wales from 1989 to 1998. We did not examine data on turtles. Each record contained the following information: date; species; reason for rescue; rescue location; injury; cause of the injury; the fate of the animal; sex (when known); and lifephase (i.e. adult or juvenile).

We analysed these data to answer the following questions:

1. What species of snakes and lizards were reported to WIRES over this period, and in what numbers?

2. Why were these animals rescued, and how did this vary among species? Within a species, did juvenile and adult specimens differ in this respect?

3. What were rates of survival from each type of injury?

4. Did the numbers of each taxon rescued, the causes for rescue, or the relative numbers of adult and juvenile animals, vary among months?

5. Did weather conditions influence the numbers of animals rescued?

6. Did temporal variation in the numbers of reptile rescues reflect reptile behaviour, or simply patterns of human activity?

Preliminary analyses indicated that patterns within the "Sydney" data were virtually identical to those from other parts of NSW. This similarity may reflect the fact that most records came from relatively large towns or cities, rather than remote rural communities. Only analyses based on the complete (NSW) data set are presented in this paper. Data on one species (bluetongue lizards, Tiliqua scincoides) have been excluded from analysis in the current paper, as this species has been the subject of a separate, more detailed study (Koenig, 1999; Koenig et al., 2001).

\section{Results}

\subsection{Numbers and composition of reptile rescues}

Over the period 1989 to 1998 , a total of $>22,000$ squamate reptiles were rescued by WIRES volunteers in New South Wales (Table 1). These specimens were identified to 38 different taxa (species or genera). Some 
of the identifications may well have been in error. For example, there were five records of "frillneck lizards" (Chlamydosaurus kingii), a species not known to occur in NSW. These animals were almost certainly Pogona spp. ("bearded dragons", frequently called "frillneck lizards" by local people), and have been allocated to this taxon in Table 1. Nonetheless, WIRES staff identify specimens carefully, are trained in identification techniques, and have access to regional field-guides that are easy to use (especially for an animal in the hand, rather than one seen in the bush). The most commonly-reported reptile taxa are generally species for which identification is particularly straightforward, because all have very distinctive features of morphology and coloration (Table 1). Thus, most identifications are likely to be accurate.

The most striking aspects of the list in Table 1 are the predominance of snakes and of one lizard species. Eastern bluetongues (Tiliqua scincoides) are the only large lizard that is common in suburban habitats (Koenig, 1999). Few other lizards were rescued (Table 1). For example, a single snake species (the red-bellied or common blacksnake, Pseudechis porphyriacus) was reported more often than all other lizard species combined (4738 versus 2182 records). Within the snakes, there was a clear tendency for large and/or dangerous taxa to be reported in much larger numbers than smaller, less dangerous species. For example, WIRES rescued >2000 carpet pythons (Morelia) and > 2000 brownsnakes (Pseudonaja), but recorded $<20$ blindsnakes (Ramphotyphlops). In many years of fieldwork in the Sydney region by one of the authors (RS), the most frequently encountered snake species have been small taxa (Ramphotyphlops, Cacophis, Cryptophis, Demansia, and Hemiaspis) that occur rarely in the WIRES data (Table 1). Similarly, small lizards (especially skinks) are much more abundant than large agamids or varanids, but the latter groups comprised the majority of reported lizard rescues. Thus, there was clearly a massive bias towards rescuing larger and more formidable species.

The other clear bias involves the morphology of lizard species that are rescued. Several of these have very short limbs and thus, superficially resemble snakes. This is true not only of virtually limbless animals (Saiphos, Lialis and Pygopus) but also of heavy-bodied skinks with relatively small limbs (Cyclodomorphus, Tiliqua). We have often been approached by concerned members of the public about "snakes" that have proven to be these short-limbed lizards.

\subsection{Causes for rescue}

The WIRES data provide a list of 19 categories for why an animal was collected. Most of these involve particular anthropogenic threats (e.g. attack by dogs or cats, poisoning, etc.). Unsurprisingly, the different causes
Table 1

Reptiles "rescued" by WIRES (Wildlife Rescue and Information Service) over the period 1989 to 1998 throughout New South Wales (NSW), and the subset of those records that came from the Sydney area

\begin{tabular}{|c|c|c|}
\hline & NSW & Sydney \\
\hline \multicolumn{3}{|l|}{ Snakes } \\
\hline \multicolumn{3}{|l|}{ Colubridae } \\
\hline Brown treesnake (Boiga irregularis) & 56 & \\
\hline Green treesnake (Dendrelaphis punctulatus) & 526 & 139 \\
\hline \multicolumn{3}{|l|}{ Elapidae } \\
\hline Death adder (Acanthophis antarcticus) & 52 & \\
\hline Copperhead (Austrelaps spp.) & 302 & \\
\hline Dwarf crown snake (Cacophis krefftii) & 30 & \\
\hline Golden crown snake (Cacophis squamulosus) & 135 & \\
\hline Small-eyed snake (Cryptophis nigrescens) & 28 & \\
\hline Yellow-faced whipsnake (Demansia psammophis) & 116 & \\
\hline White-lipped snake (Drysdalia coronoides) & 14 & \\
\hline Red-naped snake (Furina spp.) & 18 & \\
\hline Swamp snake (Hemiaspis signata) & 436 & \\
\hline Stephens banded snake (Hoplocephalus stephensii) & 2 & \\
\hline Tigersnake (Notechis scutatus) & 283 & \\
\hline Red-bellied blacksnake (Pseudechis porphyriacus) & 4738 & 1563 \\
\hline Brownsnake (Pseudonaja textilis) & 2078 & 782 \\
\hline Bandy-bandy (Vermicella annulata) & 25 & \\
\hline \multicolumn{3}{|l|}{ Pythonidae } \\
\hline Carpet python (Morelia spilota) & 2210 & 511 \\
\hline \multicolumn{3}{|l|}{ Typhlopidae } \\
\hline Blind Snakes (Ramphotyphlops spp.) & 18 & \\
\hline Total snakes & 11067 & 2995 \\
\hline
\end{tabular}

Lizards

Agamidae

Bearded dragon (Pogona spp.)

Mountain dragon (Tympanocryptis diemensis)

Jacky lizard (Amphibolurus muricatus)

Water dragon (Physignathus lesueurii)

Gekkonidae

Leaf Tail Gecko (Phyllurus platurus)

Thick tail Gecko (Underwoodisaurus milii)

Scincidae

Striped skink (Ctenotus spp.)

Cunningham's skink (Egernia cunninghami)

Land mullet (Egernia major)

Three-toed skink (Saiphos equalis)

Water skink (Eulamprus quoyii)

She-oak skink (Cyclodomorphus casuarinae)

Pink-tongue (Cyclodomorphus gerrardii)

Blotched bluetongue (Tiliqua nigrolutea)

Shingleback (Tiliqua rugosa)

Eastern bluetongue (Tiliqua scincoides)

Pygopodidae

Burton's legless lizard (Lialis burtonis)

Scaly foot (Pygopus lepidopodus)

Varanidae

Gould's monitor (Varanus gouldii)

Lace monitor (Varanus varius)

533

Total lizards

Total reptiles 
for rescue were not equally important in any of the species for which we examined data. That is, contingency-table tests enabled strong rejection of the null hypothesis of equal numbers of rescues for each cause. For these tests, we classified the cause into eight categories (to reduce the frequency of low expected numbers) as follows: attack by cat; attack by dog; car; unsuitable environment; habitat loss; attack by bird; entanglement; other. For every taxon that we tested in this way, the $P$ value was $<0.001$ (see Fig. 1).

Biologically, the more interesting question is whether the causes for rescue were similar among different taxa. A clear dichotomy was evident between snakes and lizards, especially with respect to the category of "unsuitable environment". These are the cases in which the reptile was "rescued" not because it was injured, but because local residents wanted it removed. More than $85 \%$ of rescues fall into this category for each of the main snake species, whereas the corresponding figure for lizards was much lower ( 38 to $73 \%$ ). That is, many lizards were rescued because they were injured, whereas

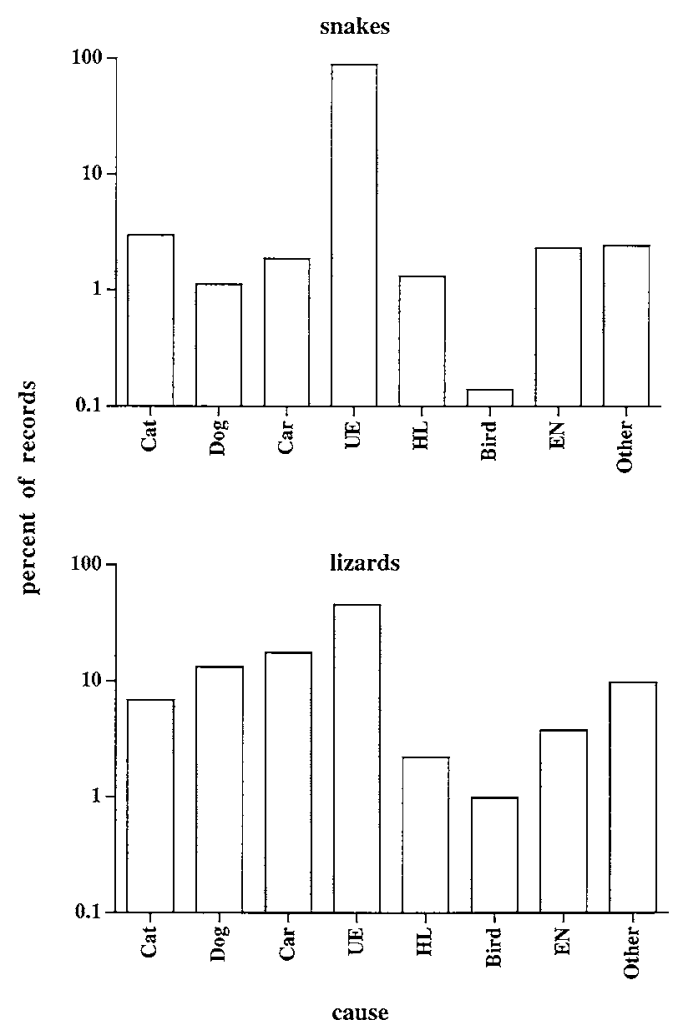

Fig. 1. Major causes of rescue by Wildlife Information and Rescue Service (WIRES) for snakes and lizards in New South Wales over the period 1989 to 1998 inclusive. See text for statistical treatment of these data. Causes for rescue include attack by domestic cats, dogs and birds; being entangled in netting ("EN"); being run over by motor vehicles ("car"); being found in an unsuitable environment ("UE"); or in an area being disturbed (habitat loss, = "HL"). Data are shown on a logarithmic scale because of very large differences in the numbers of records among different causes. most snakes were "rescued" because their presence was unwelcome (Fig. 1).

If we look more closely within each of the major groups, other differences become apparent. Comparing the relative numbers of records for each cause of rescue among the nine most commonly-reported snake species, a contingency-table test reveals highly significant differences among species $\left(\chi^{2}=1006.7,56\right.$ d.f., $\left.P<0.0001\right)$. More detailed inspection reveals that attacks by cats were more important for smaller snake species (e.g. Cacophis, Demansia, Hemiaspis) than for larger taxa (such as Morelia, Pseudechis, and Pseudonaja: see Fig. 2). A similar analysis for the five most common lizard species generated a similar result. There were significant differences among species in the relative numbers of reptiles "rescued" for different causes $\left(\chi^{2}=228.6\right.$, 16 d.f., $P<0.0001$; see Fig. 2). As in the snakes, the largest species (varanids) were generally removed because of "unsuitable environment", whereas smaller species (skinks and agamids) were often rescued after being attacked by domestic pets (Fig. 2).

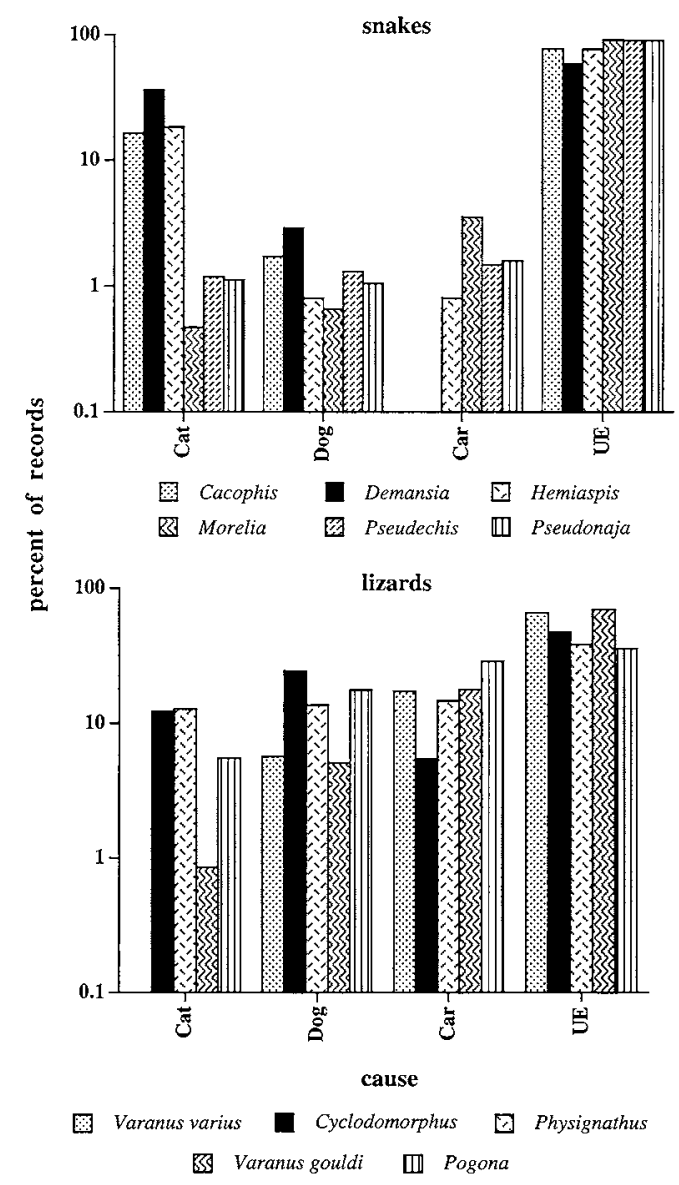

Fig. 2. Major causes of rescue by Wildlife Information and Rescue Service (WIRES) for the most commonly-recorded species of snakes and lizards. See text for statistical treatment of these data. "UE" = unsuitable environment. See Table 1 for names of the species involved (referenced by their generic name only in the Figure). 
How consistent is this association between body size and the cause for rescue by WIRES? We extracted data on adult sizes of snakes from published literature (Shine, 1994), and compared mean adult snout-vent length to the relative importance of each cause for rescue. Larger species of snakes were less likely to be brought in after being injured by domestic cats (mean SVL versus \% of records due to cat attacks: spearman rank $\rho=-0.95, P<0.01)$ but more likely to be hit by cars (spearman rank $\rho=0.69, P<0.05$ ).

If a species' body size influences its vulnerability to different kinds of injury, we might expect a similar effect within each species. That is, juvenile and adult individuals would show different patterns of causation for rescue. Analysis supports this proposition, for both snakes $\left(\chi^{2}=80.1,6\right.$ d.f., $\left.P<0.0001\right)$ and lizards $\left(\chi^{2}=15.2,6\right.$ d.f., $P<0.012$; Fig. 3$)$. As expected, attacks by domestic cats were disproportionately directed towards juvenile reptiles. Most victims rescued after being run over by motor vehicles were adults, perhaps because juvenile animals were unlikely to be noticed, or to survive this experience for long enough to appear on the WIRES database (Fig. 3).

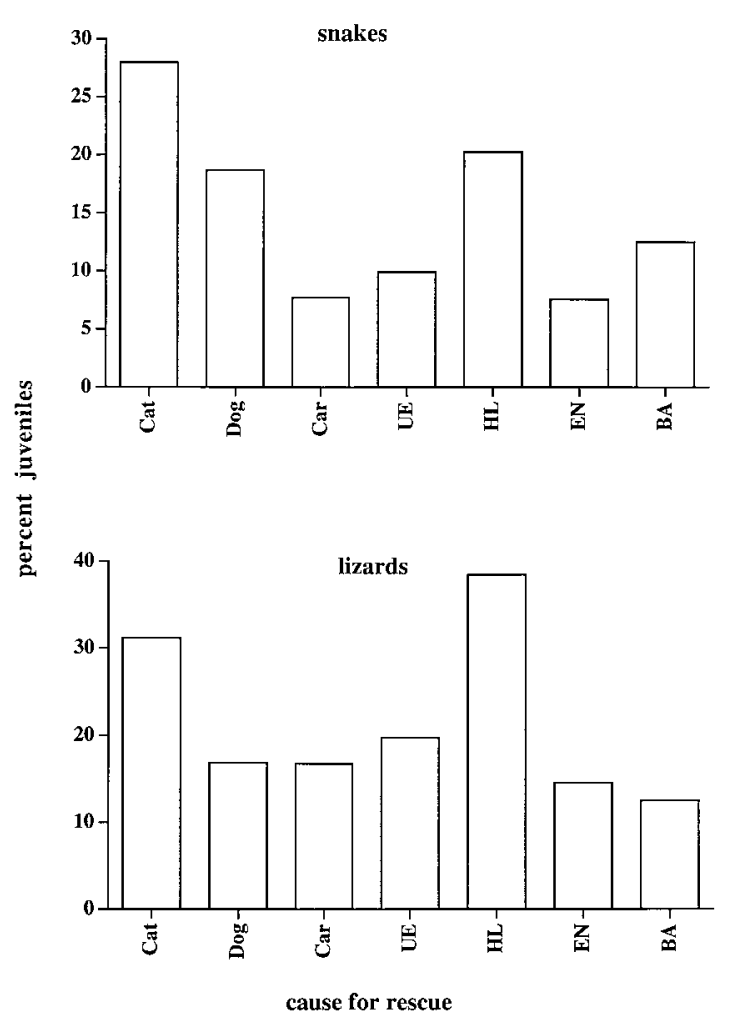

Fig. 3. Major causes of rescue by Wildlife Information and Rescue Service (WIRES) for adult versus juvenile specimens of snakes and lizards. See caption to Fig. 1 for abbreviations, and see text for statistical treatment of these data. Data are shown on a logarithmic scale because of very large differences in the numbers of records among different causes.

\subsection{Rates of survival}

Given the wide diversity among species rescued by WIRES volunteers (Table 1), and the interspecific differences in causes for such rescues (e.g. Figs. 2 and 3), it seems likely that species will also differ in their rates of survival after rescue. Analysis strongly confirms this prediction (comparing the most common species in terms of relative numbers surviving versus dying: for five species of lizards, $\chi^{2}=57.51,4$ d.f., $P<0.0001$; for eight species of snakes, $\chi^{2}=72.35,7$ d.f., $P<0.0001$ ). The proportions of animals recorded as surviving long enough to be released elsewhere ranged from $57 \%$ (Cyclodomorphus gerrardii) to 95\% (Morelia spilota). Proportions of surviving animals tended to be higher for snakes (overall mean $=93.2 \%)$ than for lizards $(72.9 \%$; $\chi^{2}=1610.7,1$ d.f., $\left.P<0.0001\right)$.

These patterns are at least partly due to differences in the causes for rescue among different taxa. Unsurprisingly, reptiles that were rescued because of injuries they had sustained were more likely to die soon afterwards than were animals that had been captured because they were found in "unsuitable habitats" (average rates of

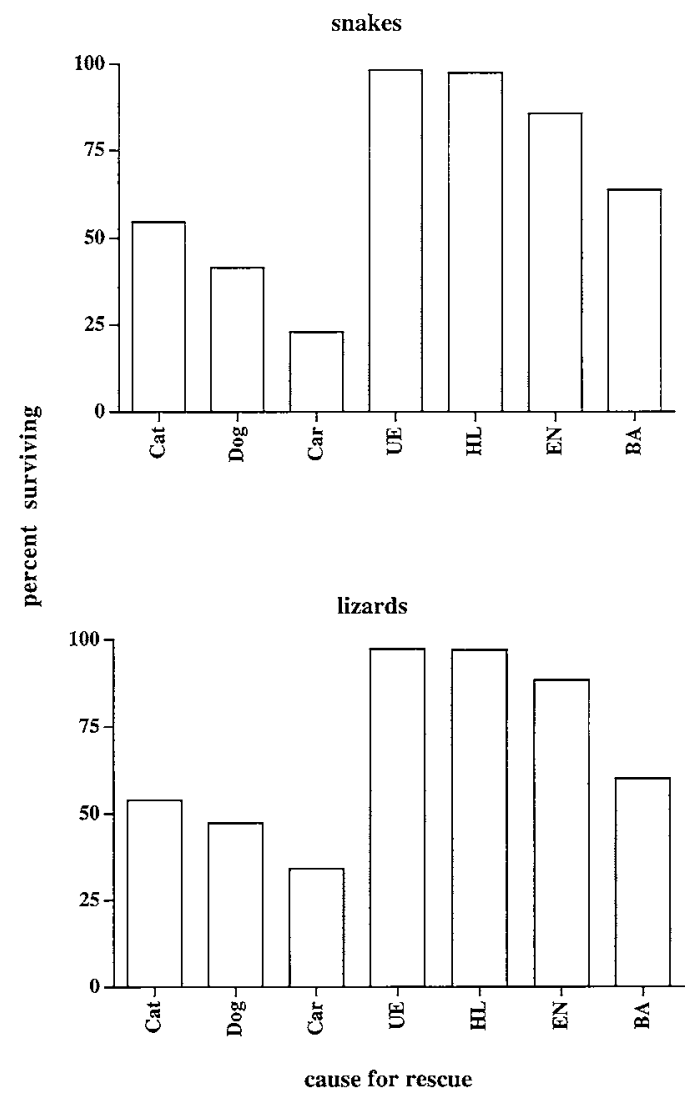

Fig. 4. Mortality rates of snakes and lizards recorded on the Wildlife Information and Rescue Service (WIRES) database as falling into the major causes for rescue. See caption to Fig. 1 for abbreviations, and see text for statistical treatment of these data. 
survival $=34 \quad$ versus $97.9 \% ; \quad \chi^{2}=7037.6, \quad 1$ d.f., $P<0.0001)$. Among the subset of animals in this latter category, survival rates were similar in lizards and snakes $(96.95$ versus $98.17 \%)$. Restricting analysis to within each species, there were highly significant differences in survival rates of animals that had been rescued for different reasons (contingency-table analyses for all common taxa, all $P<0.01$ ).

If we restrict attention to injured reptiles, survival rates differed in complex ways among causes of such
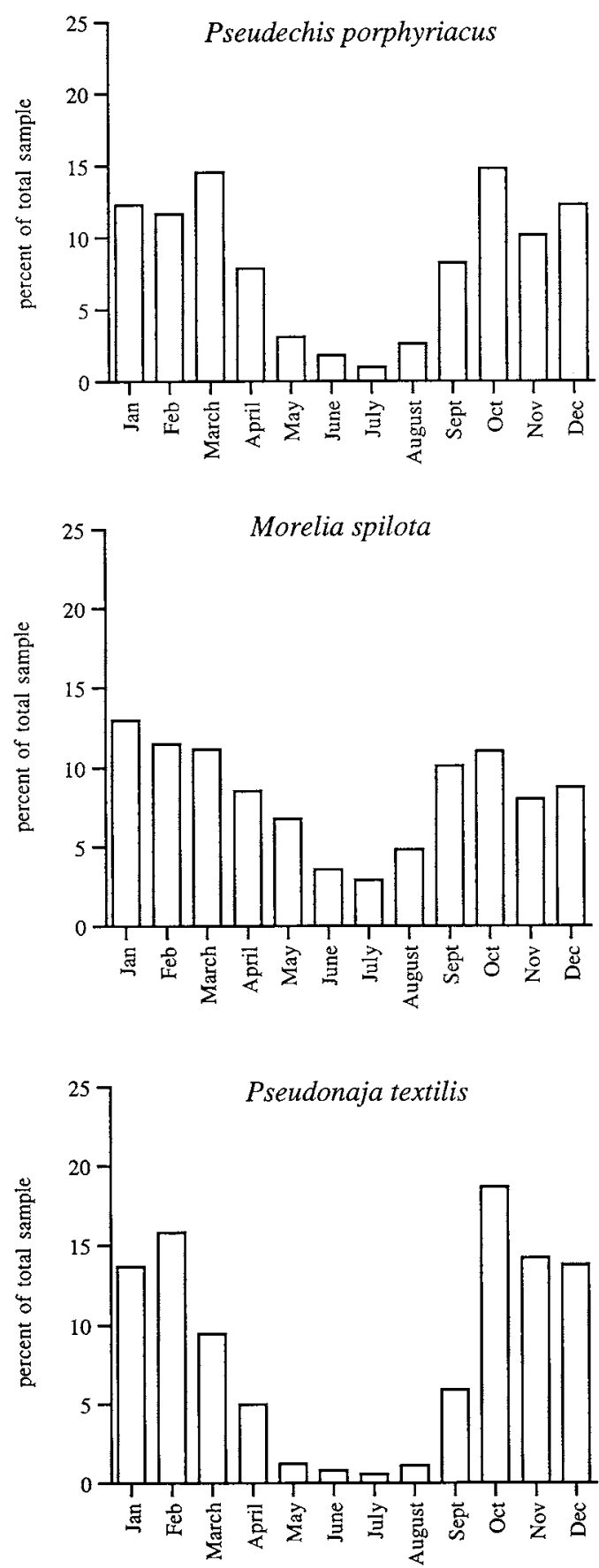

injury. Nonetheless, some general patterns were apparent. As a general rule, attacks by cats were less likely to result in the reptile's demise than were attacks by dogs (Fig. 4 ; 48.8 versus $32.9 \% ; \chi^{2}=67.88$, 1 d.f., $P<0.0001$ ). However, several species showed the reverse pattern, with higher mortality from cats than dogs (Cacophis, Pseudonaja, Physignathus). Survival of reptiles that had been run over by motor vehicles was generally low, averaging $<25 \%$ in most taxa (Fig. 4). Surprisingly, mortality rates from some sources were actually higher
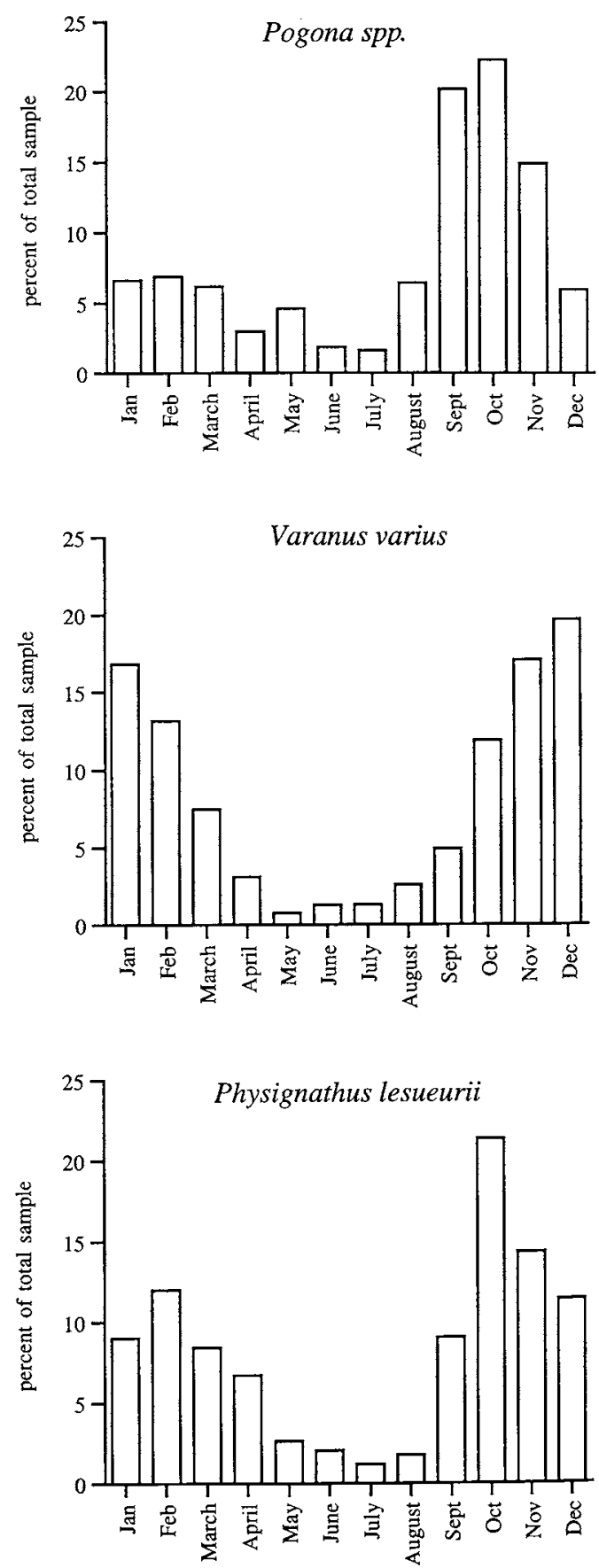

Fig. 5. Monthly distributions of the numbers of individuals of species of snakes and lizards commonly-recorded in the Wildlife Information and Rescue Service (WIRES) database. See text for statistical treatment of these data. 
for larger snake species than for smaller ones. The pattern is complex, however. Mortality rates were also low for the largest snake species (the carpet python, Morelia spilota).

\subsection{Temporal patterns in the numbers of rescues}

As one might expect from ectothermic animals living in a temperate climatic zone with substantial seasonal
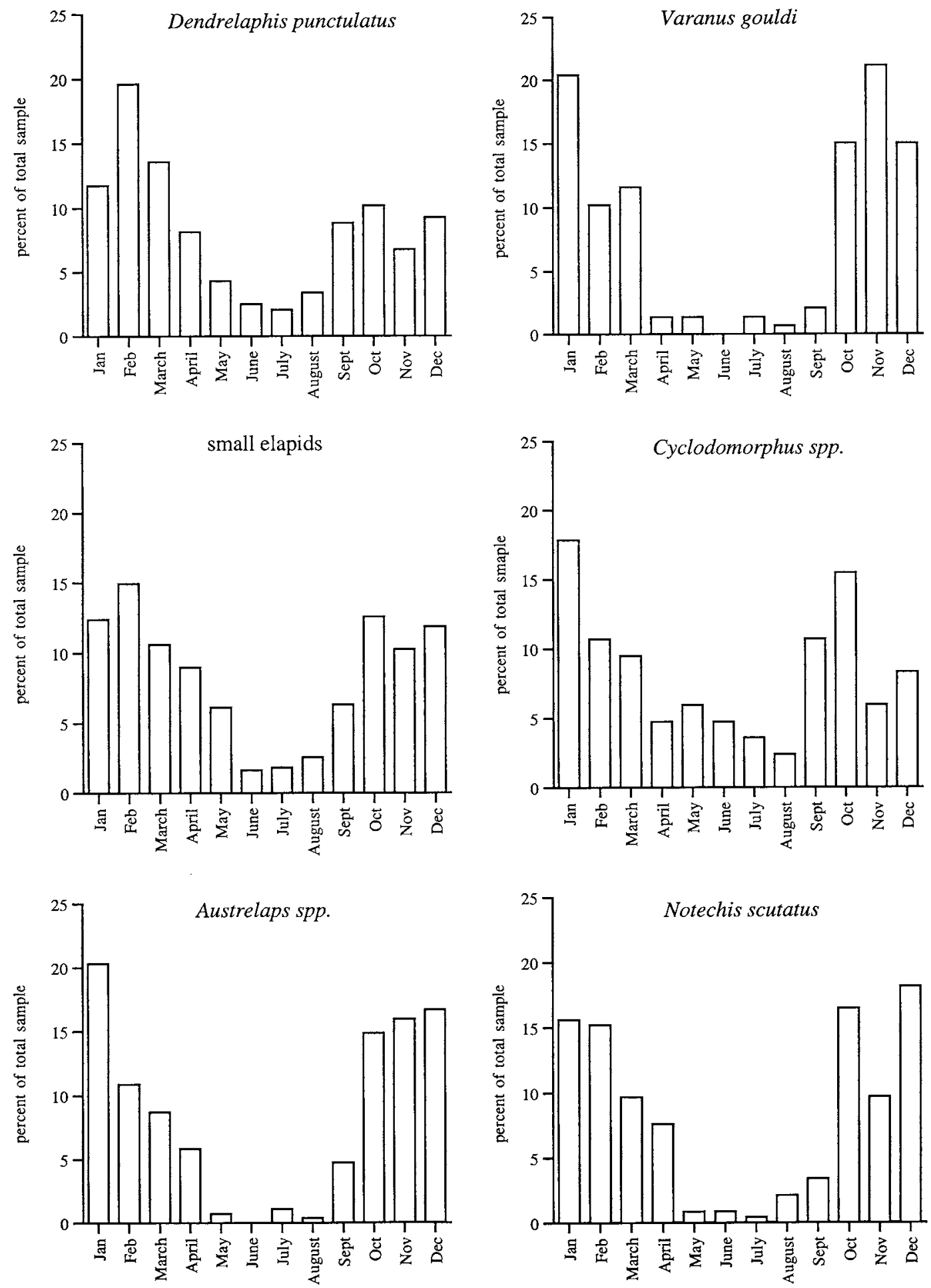

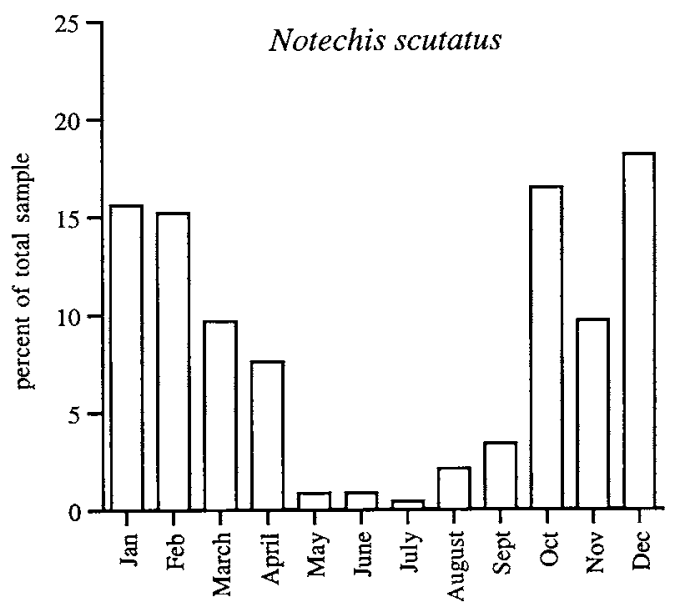

Fig. 6. Monthly distributions of the numbers of individuals of species of snakes and lizards commonly-recorded in the Wildlife Information and Rescue Service (WIRES) data-base. "Small elapids" = combined records for Cacophis spp., Hemiaspis signata, and Cryptophis nigrescens. See text for statistical treatment of these data. 
shifts in temperature, WIRES records for reptile rescues were concentrated in warmer months of the year (Figs. 5 and 6). When data for each of these species were analysed separately, the distribution of records differed from that expected under the null hypothesis of equal numbers per month $(P<0.01$ for all species). Nonetheless, the exact nature of that deviation from equal numbers differed among taxa. For example, pythons (Morelia) were frequently encountered during winter, whereas large elapids (e.g. Austrelaps, Notechis, Pseudonaja) and varanids (Varanus) were rarely encountered at these cooler times (Figs. 5 and 6).

The relative numbers of adults and juveniles within each species might also be expected to shift seasonally, because reproduction is highly seasonal over the study area (e.g. Shine, 1985). This pattern was observed in some species. For example, the proportion of juvenile bearded dragons (Pogona spp.) increased from $<10 \%$ in spring to $>30 \%$ after the hatching period in summer (comparing the four seasons, $\chi^{2}=17.03,3$ d.f., $P<0.001)$. The same kind of pattern was evident in redbellied blacksnakes (Pseudechis porphyriacus), with juveniles representing only $20 \%$ of records in spring and summer, but $>50 \%$ of records after parturition in autumn $\left(\chi^{2}=3.01,3\right.$ d.f., $\left.P<0.0001\right)$.

Causes for rescue showed little variation among seasons, especially in snakes (for which rescues from "unsuitable environment" were always dominant). Attacks by domestic cats tended-to be higher in autumn than in other seasons, and injuries due to motor vehicles peaked in spring.

\subsection{Influence of weather conditions}

Reptile activity is strongly weather-dependent, and animals that are moving may be more likely to encounter humans (Bonnet et al., 1998). Do we see any such link in the WIRES data? The correlation between weather conditions and number of rescues that emerges from broad monthly comparisons (i.e. more reptiles in warmer months: see Figs. 5 and 6 ) is consistent with this idea, but might be an indirect effect of other factors. For example, reproductive seasonality might drive this correlation, rather than any causal influence of weather per se. To test the idea that weather conditions influence rates of encounter, we need to restrict analysis to within a given season. Fig. 7 shows such an analysis for a subset of our data during autumn (March-May). The number of reptiles rescued by WIRES was higher on hotter days (for both minimum and maximum air temperature) and lower on rainy days (see Fig. 7 caption for statistical results). More detailed analysis of these patterns may reveal greater complexity; for example, capture rates declined in very hot weather (Fig. 7). Similar correlations were evident at other times of year, and the same kinds of patterns were evident when we repeated these analyses separately for the most common species.

\subsection{Artefacts due to human activity}

Associations between rates of reptile-human encounter and weather conditions (Fig. 7) or times of year (Figs. 5 and 6) might be due to shifts in activity levels of either participant in the encounter. Plausibly, temporal patterns in numbers of reptile records might tell us less about reptile ecology than about human activity. For example, more reptiles might be reported on fine days because more humans are out in their gardens, rather than because reptiles are more active.

One way to test this notion is to look at relative numbers of WIRES records for each day of the week. Human activity outdoors shows a strong weekly cycle (more activity on weekends) whereas reptile activity does not. To investigate this possibility, we compared
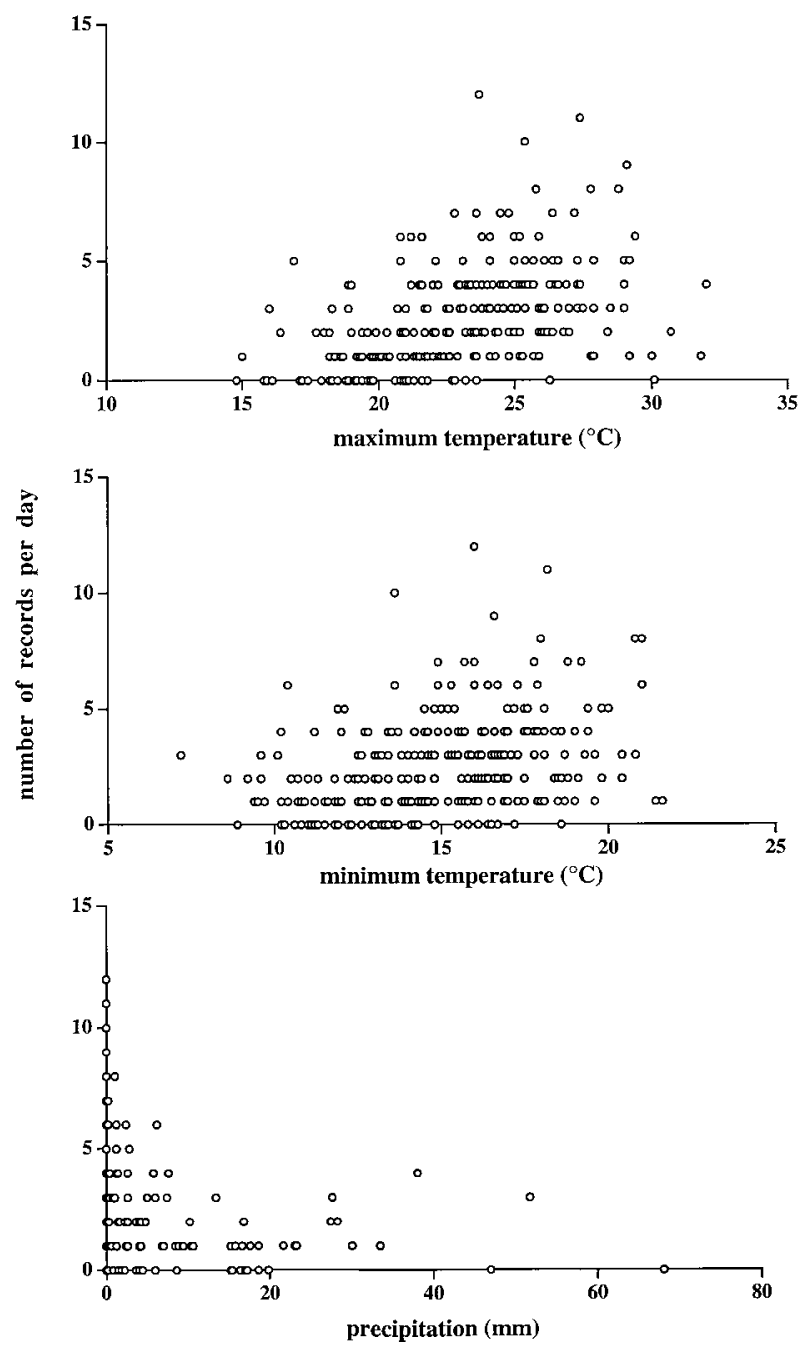

Fig. 7. The number of snakes recorded by Wildlife Information and Rescue Service (WIRES) each day in autumn (March to May, data combined over the years 1995 to 1997), compared with weather conditions that day. Each point represents data for 1 day. The number of snakes collected by WIRES was positively correlated with maximum air temperature $\left(r^{2}=0.21\right.$, d.f. $\left.=1,274, P<0.0001\right)$ and minimum air temperature $\left(r^{2}=0.11\right.$, d.f. $\left.=1,274, P<0.0001\right)$ and negatively correlated with precipitation $\left(r^{2}=0.04\right.$, d.f. $\left.=1,274, P<0.001\right)$. 


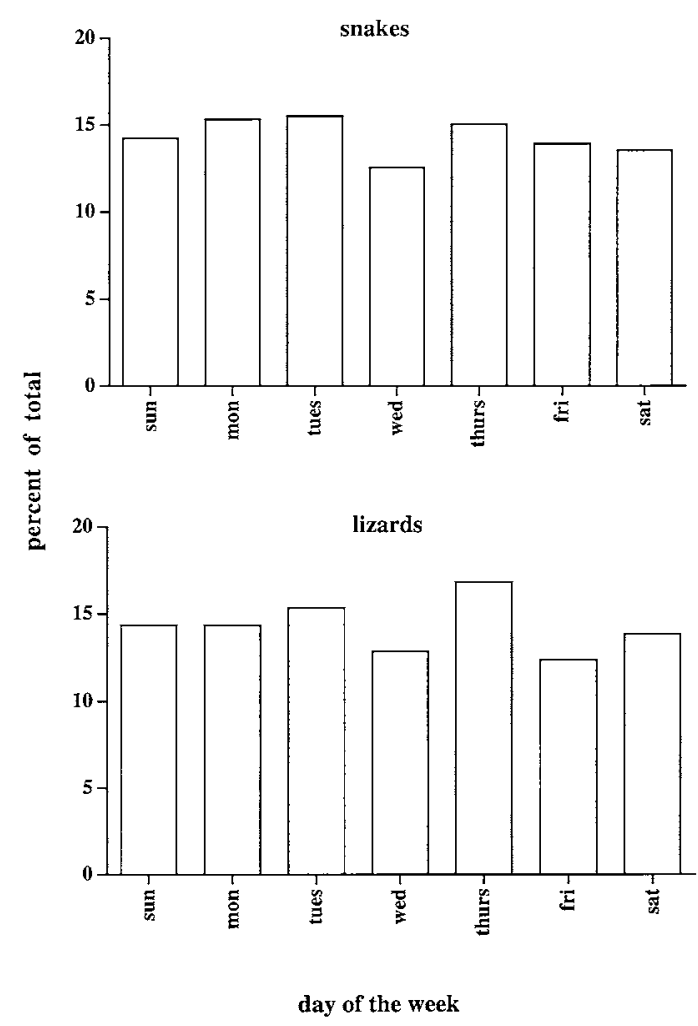

Fig. 8. Numbers of snakes (upper graph) and lizards (lower graph) recorded by Wildlife Information and Rescue Service (WIRES) on each day of the week. Data for all species combined within each category. See text for statistical treatment of these data.

numbers of rescues for each day of the week over three complete years (1995-97). Fig. 8 shows the results of this analysis, revealing that the numbers of reptiles recorded by WIRES remained high throughout all days of the week. There was no significant daily variation in the number of records reported to WIRES for either snakes $\left(\chi^{2}=5.20,6\right.$ d.f., $P=0.52)$ or lizards $\left(\chi^{2}=1.88,6\right.$ d.f., $\left.P=0.93\right)$.

\section{Discussion}

Below, we treat our analysis as a case study on the way in which "wildlife rescue" activities may be relevant to the activities of professional ecologists. As noted in Section 1, these community-based activities may have direct ecological impacts on natural populations (some negative, some positive). At the same time, records maintained by these groups contain information on topics such as the local distribution and abundance of wildlife, the nature of threats to populations, and the biological traits of poorlyknown species. We consider both of these aspects later.

\subsection{Direct ecological impact of "wildlife rescue" activities}

\subsubsection{Numbers and types of reptiles affected}

WIRES has rescued a very large number of reptiles in New South Wales over a 10-year period, especially from the region around Sydney (Table 1). Undoubtedly, many other reptiles were rescued but not entered into the database. WIRES is only one of several wildlife rescue groups in NSW, and similar activities are often performed by people who do not belong to any formal grouping (pers. obs.; Bush et al., 1995). In our own experience, most large towns and cities in Australia have some kind of arrangement whereby "problem" wildlife is identified and removed. In small towns, this may involve only a single person - but the numbers of animals involved may nonetheless be considerable. For example, one part-time snake-removalist in a semi-rural area of north-eastern NSW (Mullumbimby) translocated $>100$ snakes and lizards, at people's requests, over a 4-year period (M. Fitzgerald, pers. comm.).

Although the numbers of individual reptiles are high, the efforts of "wildlife rescuers" are strongly focused on a small number of taxa. The list of species rescued by WIRES (Table 1) bears little relationship to the relative abundances of various reptile taxa as determined by other methods (e.g. numbers in museum collections: Longmore, 1989). NSW contains 136 lizard species and 63 terrestrial snake species (Lunney et al., 2000). Of these, about 41 species of lizards and 50 species of snakes occur in the Sydney region (Griffiths, 1987; Swan, 1990). In WIRES record, only 33 taxa (16 lizards, 17 snakes) were represented by $>10$ individuals (Table 1). Thus, these rescued animals belonged to < $20 \%$ of the squamate species in NSW.

Importantly, the species that are commonly rescued comprise a highly non-random assortment in terms of body sizes and shapes. Certain kinds of animals are more likely to be rescued than others: a koala will attract more attention than a possum. Thus, "wildlife rescue" records will give an inaccurate picture of the relative numbers of encounters with these two species. Our analysis suggests a dichotomy within the species of animals rescued by WIRES. Many species (most mammals and birds?) are rescued because they are injured or otherwise thought to be in need of assistance, whereas other taxa (reptiles, and perhaps a few "problem" mammals such as brushtail possums, Trichosurus vulpecula: Statham and Statham, 1997) are removed because they are unpopular. That is, the species most intensively targeted by wildlife rescue groups may fall into two main categories: those that attract the highest regard from the public, and those that inspire the most fear. In the case of reptiles, the clear bias is for over-reporting of formidable (large and/or snake-like) species.

\subsubsection{Effect of rescue activities on mortality rates}

We know the short-term survival rates of rescued reptiles, but cannot evaluate their probable fate had they not been collected by WIRES volunteers. In general, survival rates of the animals after rescue were very high, primarily because most were "rescued" not 
because of injury, but because they were perceived as a danger to local people. The short-term survival rates of these animals may well be enhanced by WIRES activities, because many of them might have been killed if nobody was available to remove them. Similarly, survival rates of injured reptiles may have been increased by WIRES intervention. Nonetheless, mortality rates (even assessed over a brief timescale) were high for several taxa (Fig. 4).

Mortality rates were closely tied to causes for rescue. Unsurprisingly, reptiles that were run over by motor vehicles had less chance of surviving than did reptiles relocated because they were in "unsuitable habitat". Reptiles attacked by domestic pets had intermediate rates of survival, with dogs being more likely to inflict fatal injuries than cats. However, the duration of survival was not recorded, and some of the injuries sustained in cat attacks eventually may have proven fatal (G. Shea, pers. comm.). Most of the patterns with respect to mortality fit well with intuition and experience. One paradoxical result was that rates of survival after injury were lower for large venomous (elapid) snakes than for smaller snake species, or large pythons. This result may reflect a bias in human behaviour. In the case of large venomous species, moderately injured animals would be difficult to capture without serious risk. If most of the collected large elapids were either uninjured, or very badly injured, we would see the pattern that is actually observed.

\subsubsection{Effects of translocation}

Paradoxically, the high survival rates of reptiles collected by WIRES volunteers may be a cause for concern in terms of conservation. Many reptiles (Table 1) are translocated to areas subjectively judged to be "suitable habitat". Most reptiles (especially snakes) in the WIRES data-base were uninjured, and removed simply so that they could be released elsewhere. WIRES volunteers release such animals in areas of natural habitat within the species' natural range, away from obvious human influence, and preferably $<20 \mathrm{~km}$ from the site of capture (M. Cooper, pers. comm.). This procedure may have several effects:

1. Survival rates of translocated individuals. - We do not know the fate of these animals, but other studies on the eventual fate of translocated reptiles are not encouraging. Radio-tracked relocated rattlesnakes tended to wander over wider areas, and experienced lower survival rates, than did resident animals (Nowak, 1997; Reinert and Rupert, 1999).

2. Spread of pathogens. - Some proportion of the reptiles captured in suburbia are undoubtedly escaped pets, that have spent unknown periods of time in captivity. Release of such animals into the wild may pose a significant threat of introducing serious diseases into wild populations (Smith et al., 1998; Berish et al., 2000).
3. Introduction of novel genetic material. - Introducing new genes may substantially enhance the long-term viability of highly inbred populations, including those that have been isolated by anthropogenic influences (Madsen et al., 1999). However, such genetic manipulations may also compromise the genetic distinctiveness of local populations.

4. Predator-prey biology. - WIRES mostly translocated large predators, especially snakes (Table 1). Like all top predators, such animals tend to be relatively rare and thus, their translocation may have ecological impacts on both the "donor" and "recipient" areas.

The importance of such effects will depend upon the conservation status of the species involved. Two of the snake species translocated by WIRES (Acanthophis antarcticus and Hoplocephalus stephensii) are listed as being of significant conservation concern (Cogger et al., 1993). Many specimens of other more common species presumably came from or were transferred to isolated fragments of habitats in highly-disturbed regions, and thus may be of conservation relevance also.

\subsection{Acquisition of data}

\subsubsection{Information on local distribution and abundance of urban wildlife}

Can the WIRES data tell us anything useful about this topic, given the massive biases inherent in the selection of individuals to be rescued? These biases mean that we cannot conclude anything about relative numbers of taxa that differ in ways likely to influence their priority for rescue - for example, small versus large animals, or lizard species that do versus do not resemble snakes. However, the data may nonetheless be useful if comparisons are made only within groups that resemble each other in these attributes. Additionally, such data may confirm the occurrence of a particular rare species within a given area (although the possibility of prior translocation remains significant). Perhaps most importantly, analysis of such databases through time may indicate changes in relative numbers of taxa through time. This inference is itself sensitive to other factors, such as changes in public attitudes towards particular types of wildlife. However, it may provide larger sample sizes (and hence, more robust inferences) than will generally be the case for studies on rare or threatened species. So long as comparisons are restricted to within "similar" taxa, or within the same taxon over time, wildlife-rescue data-sets may be informative about patterns of local distribution and abundance. Thus, for example, declines in vertebrate species richness with increasing urbanisation may well be detectable from long-term records of wildlife rescue groups. 
Large venomous snakes provide a special case, because they are probably the group most likely to be reported to organisations such as WIRES. Hence, rates of reporting may provide a reliable indication of patterns of occurrence through time and space. This information is of obvious value in terms of evaluating the potential risk of snakebite.

\subsubsection{Information on the nature of threats to urban wildlife}

The databases of wildlife-rescue groups allow us to quantify the relative numbers of different species brought in for various reasons. Thus, for example, we can identify domestic cats as a significant mortality source for small reptile species and for the juveniles of some larger taxa (Figs. 2-4). In contrast, the significance of vehicular accidents cannot be established from the data, because most reptiles run over by vehicles are likely to die before they can be collected. In the same way, cats and dogs consume many small reptiles such as garden skinks of the genus Lampropholis, undoubtedly the most abundant reptiles in suburban Sydney (Griffiths, 1987). These species are killed in greater numbers by domestic pets and motor vehicles than any other taxa, yet they are not represented in WIRES records at all (Table 1). Presumably, people do not bother to report such small animals to WIRES. As a result, the database on causes of rescues may tell us little about the numerical importance of different causes of mortality for particular species. Nonetheless, longitudinal studies (sources of rescue and mortality of the same species in the same region, followed through an extensive time period) might reveal changing patterns in mortality sources.

Injuries by domestic pets (especially cats) were concentrated on smaller species of reptiles, and on juveniles rather than adults. Presumably, pets were unable or unwilling to tackle larger prey. The size-dependence of factors such as cat predation is relevant to conservation issues. The impact of predation on population viability depends not only upon the actual numbers of animals killed, but also on the concentration of that offtake on particular age or sex classes (Caughley and Sinclair, 1994). For example, the high mortality due to motor vehicles in spring is probably concentrated on matesearching adult males, and may thus have less impact on population viability than other sources of mortality (Bonnet et al., 1998).

\subsubsection{Information on the biological attributes of wildlife species}

For many animal species, even those that penetrate into suburbia, there is little reliable information on topics such as the ways in which short- or long-term variation in weather conditions affect activity patterns. This dearth of information holds true for almost all of the reptile species listed in Table 1 . Given the strong biases inherent in the activities of a group such as WIRES, what can we learn from their records? As noted above, comparisons that are restricted to trends within a single species, or a set of similar taxa, may be informative. The strongest comparisons in this respect involve temporal variation in rates of collection and in the recorded causes of mortality.

Interspecific differences in the seasonal timing of records (as in the present study: e.g. pythons versus large elapid snakes) presumably reflect underlying biological attributes of these taxa. All of the reptile species showed seasonal patterns of activity (as judged by the numbers of records: Figs. 5 and 6) and were more likely to be collected in warm, dry weather (Fig. 7). The lack of strong "day of the week" effects on rates of collection (Fig. 8) suggests that artefacts due to human activity are relatively minor, and thus not responsible for these overall temporal patterns. Thus, this is an aspect of reptile biology for which "wildlife rescue" records may provide reliable and extensive data. Previous analyses of capture rates by birds of prey have documented a similar increase in vulnerability of reptiles in warm weather (Christian and Tracy, 1981; Peterson et al., 1993). Particularly for poorly-known species, information on the seasonality or weather correlates of capture rates may provide a useful basis from which to predict the optimal times for searching for such animals, and the times at which these creatures are most vulnerable to anthropogenic disturbance. Similar seasonal biases have been documented in "accidental" human-induced mortality of other animal species (e.g. birds killed in collisions with fences; roadkilled mammals: Baines and Summers, 1997; Philcox et al., 1999).

Significant differences among species in the seasonality of interactions with humans are also often reported (see earlier references) and in the present case, are readily interpretable in the light of previous research. For example, carpet pythons (Morelia spilota) showed less seasonality in capture rates than did other species of large snakes, with frequent records even during winter (Fig. 5). Radio-tracked pythons in the Sydney suburbs frequently moved to houses at this time, to spend winter in protected spaces under the roof (Slip and Shine, 1988). Such animals are thus likely to be encountered whenever the householder ventures into this space. In contrast, brownsnakes (Pseudonaja), tigersnakes (Notechis) and copperheads (Austrelaps) were encountered almost exclusively in warmer months of the year (Figs. 5 and 6). Again, telemetry clarifies this result. Brownsnakes are fast-moving diurnal snakes which are active only when they can attain high body temperatures; they spend winter inactive inside burrows (Whitaker, 1999). Tigersnakes and copperheads are restricted to relatively cool climates, where the severe winter impedes snake activity (e.g. Shine, 1979). Hence, people rarely encountered these snakes in cool weather. 
Seasonality can also reflect temporal shifts in the body-size distribution of the population. For example, cat attacks were concentrated in summer because smaller (younger) specimens comprise a higher proportion of the total reptile population in the period immediately after birth or hatching. Neonates tend to disperse during this period also, making them even more visible and more vulnerable to attack.

In summary, our study supports the notion that "wildlife rescue" activities are relevant to conservation biology, and warrant further attention from ecologists. On the negative side, the wildlife-based activities of animal welfare groups (especially translocation) may threaten the viability or genetic integrity of some populations. On the positive side, data gathered by these groups may provide insight into the biology of native fauna, local patterns of distribution and abundance, and the nature of interactions between fauna and people. Sample sizes for many species are so large, and patterns so strong, that they enable confident statistical rejection of null hypotheses. Some of those patterns result from aspects of human behaviour (e.g. widespread fear of snakes, and failure to distinguish between snakes and lizards). Other aspects result from biological characteristics of the wildlife species involved. Separating these influences, and working out how we can best use the information embodied in "wildlife rescue" data, remains a substantial challenge for researchers. It also represents a unique opportunity for constructive dialogue between researchers and "wildlife carers": two groups of people who share a passion for wildlife, but often disagree about the ways in which natural populations should be managed and conserved.

\section{Acknowledgements}

We thank the staff at WIRES (especially, Robyn Auld) for their generous provision of information. We also acknowledge our gratitude to the many WIRES volunteers who helped to gather these extensive data, and who were prepared to go to considerable inconvenience (and sometimes, danger) to rescue reptiles. S. Ormerod's suggestions greatly improved the manuscript. The work was funded by the Australian Research Council.

\section{References}

Baines, D., Summers, R.W., 1997. Assessment of bird collisions with deer fences in Scottish forests. Journal of Applied Ecology 34, 941948.

Berish, J.E., Wendland, L.D., Gates, C.A., 2000. Distribution and prevalence of upper respiratory tract disease in gopher tortoises in Florida. Journal of Herpetology 34, 5-12.
Bonnet, X., Naulleau, G., Shine, R., 1998. The dangers of leaving home: dispersal and mortality in snakes. Biological Conservation $89,39-50$.

Bush, B., Maryan, B., Browne-Cooper, R., Robinson, D., 1995. A Guide to the Reptiles and Frogs of the Perth Region. University of Western Australia, Perth, WA.

Caughley, G., Sinclair, A.R.E., 1994. Wildlife Ecology and Management. Blackwell Scientific Publications, Boston.

Christian, K.A., Tracy, R., 1981. The effect of the thermal environment on the ability of hatchling land iguanas to avoid predation during dispersal. Oecologia 49, 218-223.

Cogger, H.G., Cameron, E.E., Sadlier, R.A., Eggler, P., 1993. The Action Plan for Australian Reptiles. Australian Nature Conservation Agency, Canberra, ACT.

Griffiths, K., 1987. Reptiles of the Sydney Region. Three Sisters Productions, Winmalee, NSW.

Kaplan, G., 1999. The role of the public in wildlife research. In: Mellor, D., Monamy, V. (Eds.), The Use of Wildlife for Research. ANZCCART, Glen Osmond, SA, pp. 34-44.

Koenig, J., 1999. Ecology of a Suburban Skink: Tiliqua scincoides, the Backyard Bluetongue. Honours thesis, Biological Sciences, University of Sydney, NSW.

Koenig, J., Shine, R., Shea, G., 2001. The dangers of life in the city: patterns of activity, injury and mortality of bluetongue lizards (Tiliqua scincoides) in suburban Sydney. Journal of Herpetology (in press).

Longmore, R., 1989. Atlas of Elapid Snakes of Australia. Australian Fauna and Flora Series, Canberra, ACT.

Lunney, D., Curtin, A., Ayers, D., GCogger, H.G., Dickman, C.R., Maitz, W., Law, B., Fisher, D., 2000. The threatened and nonthreatened native vertebrate fauna of New South Wales: status and ecological attributes. New South Wales National Parks and Wildife Service, Environmental and Heritage Monograph Series, Hurstville, NSW.

Madsen, T., Shine, R., Olsson, M., Wittzell, H., 1999. Restoration of an inbred adder population. Nature 402, 34-35.

Nowak, E.M., 1997. Rattlesnake relocation at Montezuma Castle National Monument. Final Report to the Southwest Parks and Monuments Association, Flagstaff, Arizona.

Peterson, C.R., Gibson, A.R., Dorcas, M.E., 1993. Snake thermal ecology: the causes and consequences of body-temperature variation. In: Seigel, R.A., Collins, J.T. (Eds.), Snakes: Ecology and Behavior. McGraw-Hill, New York, pp. 241-314.

Philcox, C.K., Grogan, A.L., Macdonald, D.W., 1999. Patterns of otter Lutra lutra road mortality in Britain. Journal of Applied Ecology 36, 748-762.

Reinert, H.K., Rupert, R.R.J., 1999. Impacts of translocation on behavior and survival of timber rattlesnakes, Crotalus horridus. Journal of Herpetology 33, 45-61.

Shine, R., 1979. Activity patterns in Australian elapid snakes (Squamata: Serpentes: Elapidae). Herpetologica 35, 1-11.

Shine, R., 1985. Reproductive biology of Australian reptiles: a search for general patterns. In: Grigg, G.C., Shine, R., Ehmann, H. (Eds.), Biology of Australasian Frogs and Reptiles. Royal Zoological Society of New South Wales, Sydney, pp. 297-303.

Shine, R., 1994. Allometric patterns in the ecology of Australian snakes. Copeia 1994, 851-867.

Slip, D.J., Shine, R., 1988. Habitat use, movements and activity patterns of freeranging diamond pythons, Morelia s. spilota (Serpentes: Boidae): a radiotelemetric study. Australian Wildlife Research 15, 515-531.

Smith, R.B., Seigel, R.A., Smith, K.R., 1998. Occurrence of upper respiratory tract disease in gopher tortoise populations in Florida and Mississippi. Journal of Herpetology 32, 426-430.

Statham, M., Statham, H.L., 1997. Movements and habits of brushtail possums (Trichosurus vulpecula Kerr) in an urban area. Wildlife Research 24, 715-726. 
Swan, G., 1990. A Field Guide to the Snakes and Lizards of New South Wales. Three Sisters Productions, Winmalee, NSW.

Underhill, L.G., Bartlett, P.A., Baumann, L., Crawford, R.J.M., Dyer, B.M., Gildenhuys, A., Nel, D.C., Oatley, T.B., Thornton, M., Upfold, L., Williams, A.J., Whittington, P.A., Wolfaardt, A.C., 1999. Mortality and survival of African penguins Spheniscus demersus involved in the Apollo Sea oil spill: an evaluation of rehabilitation efforts. Ibis 141, 29-37.

Whitaker, P.B., 1999. Behavioural Ecology of the Eastern Brownsnake, Pseudonaja textilis (Elapidae), and implications for human envenomation. PhD thesis, University of Sydney, NSW. 